\title{
Actuator saturation and anti-windup compensation in event-triggered control
}

\author{
Georg Alexander Kiener • Daniel Lehmann • \\ Karl Henrik Johansson
}

Received: 13 April 2012 / Accepted: 10 September 2012

(C) Springer Science+Business Media, LLC 2012

\begin{abstract}
Event-triggered control aims at reducing the communication load over the feedback link in networked control systems by sending information only if certain event conditions, which guarantee a desired control performance, are satisfied. This article investigates the consequences of actuator saturation on the behavior of the event-triggered control loop in terms of its stability and information exchange. Stability properties are derived using linear matrix inequalities (LMIs) which show how the stability of the event-triggered control loop depends on the selection of the event threshold. Moreover, it is shown that a lower bound on the minimum inter-event time exists being likewise affected by the event threshold. As actuator saturation might severely degrade the performance of the event-triggered closedloop system, the scheme is extended by incorporating an anti-windup mechanism in order to overcome this problem. The results are illustrated by simulations and experiments.
\end{abstract}

Keywords Networked control systems • Event-triggered control • Stability • Actuator saturation • Anti-windup compensation • LMIs

This work was supported by the VINNOVA project WiComPI, the Knut and Alice Wallenberg Foundation, the Swedish Research Council, and the HYCON2 EU project.

G. A. Kiener · D. Lehmann $(\bowtie) \cdot$ K. H. Johansson

Automatic Control Laboratory, KTH Royal Institute of Technology,

10044 Stockholm, Sweden

e-mail: dlehmann@kth.se

G. A. Kiener

e-mail: gakiener@kth.se

K. H. Johansson

e-mail:kallej@kth.se 


\section{Introduction}

\subsection{Event-triggered control}

In networked control systems (NCS), the communication between the sensors, the controller and the actuators is realized over a digital communication medium. Compared to the traditional point-to-point architecture of a wired communication, a digital network offers several benefits with respect to lower costs, a simplified installation and maintenance. As a central feature, it additionally allows an almost unlimited flexibility in setting up and changing the required communication infrastructure. Hence, the communication links between the relevant nodes of the network can be simply adapted to the current needs.

However, the communication network has a considerable influence on the loop performance since its load affects the quality of service by inducing delays or packet losses which degrade the system performance or even cause the instability of the control loop. To avoid this situation, communication and control need to be investigated with respect to their interaction (Bemporad et al. 2010; Lehmann 2011; Nair et al. 2007; Zhang et al. 2001).

In this context, the analysis of event-triggered control has gained attention by considering event-triggered control as a means to reduce the communication load of the network by invoking an information exchange only when certain event conditions implemented by an event generator are met (Årzén 1999; Åström and Bernhardsson 1999; Bemporad et al. 2010; Tipsuwan and Chow 2003). The main aim to be reached by this feedback structure is the adaptation of the communication among the components of the feedback loop to the current requirements preserving a desired performance of the closed-loop system. In fact, by reducing the information exchange to the minimum communication that is necessary to ensure the required system performance, an overload of the digital communication network can be avoided.

The event-triggered control loop as considered in this article is depicted in Fig. 1. It consists of

- the plant with state $\boldsymbol{x}_{p}(t)$, output $\boldsymbol{y}(t)$, exogenous disturbance $\boldsymbol{d}(t)$ and input $\tilde{\boldsymbol{u}}(t)$ subject to actuator limitations,

- an event generator which invokes a communication whenever the plant output $\boldsymbol{y}(t)$ satisfies certain event conditions,

- and the controller with state $\boldsymbol{x}_{c}(t)$ which is used together with the reference input $\boldsymbol{w}(t)$ and the information $\boldsymbol{y}\left(t_{k}\right)$ to continuously produce the control input $\boldsymbol{u}(t)$.

The controller and the sensor node are connected by means of a digital network. Only at event times $t_{k}(k=0,1,2, \ldots)$ determined by the event generator, the measured plant output $\boldsymbol{y}\left(t_{k}\right)$ is sent from the event generator towards the controller which is indicated by the dashed lines. The solid lines indicate continuous-time signals.

\subsection{Literature review}

Discrete-event systems and hybrid systems have a major impact on several control application domains whose event-driven nature often results from the technology 
Fig. 1 Event-triggered control loop subject to actuator limitations

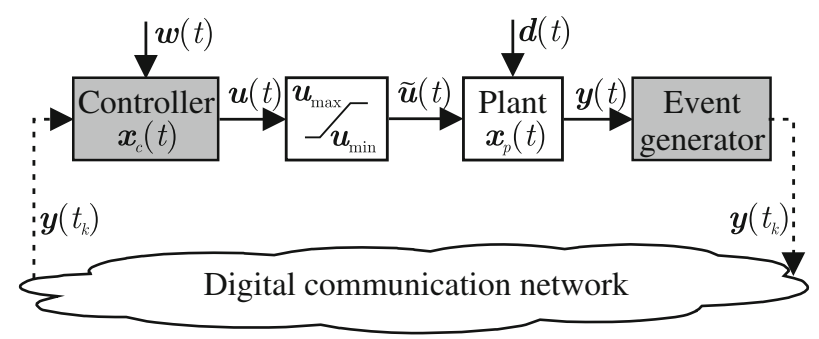

used, e.g., digital computers, manufacturing facilities and communication networks (Cassandras and Lafortune 2008; Lunze and Lamnabhi-Lagarrigue 2009).

In the last decade, there has been an additional interest in studying classical feedback control under event-driven sampling, also called asynchronous sampling, event-based sampling or event-triggered sampling ( $⿱$ Åström and Bernhardsson 1999; Heemels et al. 1999, 2008; Tabuada 2007). Most of this work is motivated by networked control systems in which the information exchange is realized over a resource-limited wireless communication network (Årzén 1999; Baillieul and Antsaklis 2007; Bemporad et al. 2010).

However, up to now most of the approaches dealing with event-triggered control, e.g., by Anta and Tabuada (2010), Cervin and Henningsson (2008), Heemels et al. (2008), Lunze and Lehmann (2010) and Wang and Lemmon (2009), consider a simple proportional controller. To make this control scheme more attractive for practical applications, there is a recent interest in considering more involved event-triggered controllers, in particular, event-triggered PID control. In this context, it has been shown by Årzén (1999), Otanez et al. (2002) and Vasyutynskyy and Kabitzsch (2006) by simulations that event-triggered PID control is able to significantly reduce the computational and communication effort while only slightly degrading the control performance.

A model-based approach to event-triggered PI control has been presented by Lehmann and Lunze (2011). It has been shown that the scheme proposed guarantees setpoint tracking for constant exogenous signals. Additionally, it provides a theoretical framework to analyze the stationary behavior of the event-triggered control loop for time-varying reference and disturbance signals. However, the scheme proposed is computationally demanding as it requires to run the model used for producing the control input $\boldsymbol{u}(t)$ both on the actuator and on the sensor node.

Donkers and Heemels (2010) considered a very general dynamic event-triggered controller. By using an extended event-generating mechanism which simultaneously monitors the evolution of the measured output $\boldsymbol{y}(t)$ and the control input $\boldsymbol{u}(t)$, the behavior of the event-triggered control loop was analyzed in terms of its stability and $\mathcal{L}_{\infty}$ properties resulting in LMI conditions.

Two general problems of event-triggered PID control are the sticking effect and large stationary oscillations (see Årzén 1999; Vasyutynskyy and Kabitzsch 2006). To overcome these problems two adaptations of the event-triggered control loop have been proposed by Tiberi et al. (2012). By using a modified event condition based on the control input $\tilde{\boldsymbol{u}}(t)$ and by adapting the integrator part of the event-triggered PI-control loop considered, it has been shown that for stable first-order systems oscillations can be avoided and setpoint tracking is guaranteed. 
However, it is well known from continuous-time control that by using a PI controller the closed-loop behavior might be significantly deteriorated whenever the actuators saturate due to physical or safety constraints (Åström and Hägglund 1995; Tarbouriech et al. 2011; Zaccarian and Teel 2011). Even though actuator saturation almost always occurs in practical applications, its effect on event-triggered control has only been studied by Lehmann and Johansson (2012) and Lehmann et al. (2012). It has been shown that actuator saturation has severe consequences with respect to the behavior of the event-triggered PI-control loop which depend on the selection of the event threshold.

\subsection{Contributions of this article}

The contributions of this article are the following:

1. It provides stability conditions based on LMIs which lead to stability regions for the event-triggered control loop (Theorem 1, Corollary 1).

2. It derives lower bounds on the minimum inter-event time showing that Zeno behavior can be excluded (Theorem 2, Corollary 2).

3. It extends the control loop by incorporating a static anti-windup mechanism in order to overcome a potential performance degradation caused by actuator saturation (Corollary 3 ).

4. It illustrates the theoretical results by simulations and experimental results showing how the stability regions are affected by the event threshold and how the anti-windup mechanism improves the results.

This article extends the results obtained in the conference papers (Lehmann and Johansson 2012; Lehmann et al. 2012), which are restricted to event-triggered PI control with full state measurement, to the situation, where a more general dynamical controller is used which might have access to only partial state information.

The remainder of this article is organized as follows. Basic notations are introduced in Section 2. The effect of saturating actuators on the stability and communication properties of the event-triggered control loop is studied in Section 3. In Section 4, the previous results are extended by incorporating a static anti-windup mechanism. Finally, Section 5 illustrates the theoretical results by simulations and experiments.

\section{Preliminaries}

\subsection{Notation}

A scalar is denoted by italic letters $(x \in \mathfrak{R})$, a vector by bold italic letters $\left(\boldsymbol{x} \in \mathfrak{R}^{n}\right)$, a matrix by bold capital letters $\left(\boldsymbol{A} \in \mathfrak{R}^{n \times n}\right)$ with $\boldsymbol{I}_{n}$ the identity matrix of size $n$ and a signal at time $t \in \mathfrak{R}_{+}$by $\boldsymbol{x}(t)$, where $\boldsymbol{x}_{0}$ describes the initial signal value at time $t=0$.

The $i$-th element of a vector $\boldsymbol{x}$ is denoted by $x_{(i)}$, the $i$-th row or column of a matrix $\boldsymbol{A}$ by $\boldsymbol{A}_{(i)}$ and the transpose of a matrix or vector by $(\star)^{T}$. Symmetric matrices of the form $\left[\begin{array}{ll}\boldsymbol{A} & \boldsymbol{B}^{T} \\ \boldsymbol{B} & \boldsymbol{C}\end{array}\right]$ are abbreviated by $\left[\begin{array}{ll}\boldsymbol{A} & \star \\ \boldsymbol{B} & \boldsymbol{C}\end{array}\right]$. Furthermore, the absolute value of a scalar 
is denoted by $|x|$, the Euclidean vector norm by $\|\boldsymbol{x}\|$ and the induced matrix norm by $\|\boldsymbol{A}\|$.

$\boldsymbol{A} \succ 0(\boldsymbol{A} \succeq 0)$ means that the matrix $\boldsymbol{A}$ is positive definite (positive semidefinite) and $\boldsymbol{A} \prec 0(\boldsymbol{A} \preceq 0)$ indicates that the matrix $\boldsymbol{A}$ is negative definite (negative semidefinite). The trace of a matrix $\boldsymbol{A}$ is denoted by $\operatorname{trace}(\boldsymbol{A})$.

\subsection{Plant description}

The plant considered in this article is described by the continuous-time state-space model

$$
\begin{aligned}
\dot{\boldsymbol{x}}_{p}(t) & =\tilde{\boldsymbol{A}} \boldsymbol{x}_{p}(t)+\tilde{\boldsymbol{B}} \tilde{\boldsymbol{u}}(t)+\tilde{\boldsymbol{B}}_{D} \boldsymbol{d}(t), \quad \boldsymbol{x}_{p}(0)=\boldsymbol{x}_{p 0} \\
\boldsymbol{y}(t) & =\tilde{\boldsymbol{C}} \boldsymbol{x}_{p}(t),
\end{aligned}
$$

where $\boldsymbol{x}_{p} \in \mathfrak{R}^{n_{p}}$ denotes the state vector, $\tilde{\boldsymbol{u}} \in \mathfrak{R}^{m}$ is the control vector, $\boldsymbol{y} \in \mathfrak{R}^{r}$ is the measured output vector and $\boldsymbol{d} \in \mathfrak{R}^{q}$ represents disturbances at the plant which are assumed to be bounded according to

$$
\boldsymbol{d} \in \mathcal{V}_{D}=\left\{\boldsymbol{d} \in \mathfrak{R}^{q}: \boldsymbol{d}^{T} \boldsymbol{Q}_{D} \boldsymbol{d} \leq \epsilon_{D}^{-1}\right\}, \quad \boldsymbol{Q}_{D}=\boldsymbol{Q}_{D}^{T} \succ 0, \quad \epsilon_{D}>0 .
$$

$\tilde{\boldsymbol{A}}, \tilde{\boldsymbol{B}}, \tilde{\boldsymbol{B}}_{D}$ and $\tilde{\boldsymbol{C}}$ are real matrices of appropriate dimensions. In the following it is assumed that the pair $(\tilde{\boldsymbol{A}}, \tilde{\boldsymbol{B}})$ is controllable and the pair $(\tilde{\boldsymbol{A}}, \tilde{\boldsymbol{C}})$ is observable.

The plant input $\tilde{\boldsymbol{u}}(t)$ is given by $\tilde{\boldsymbol{u}}(t)=\operatorname{sat}(\boldsymbol{u}(t))$, where $\operatorname{sat}(\star)$ represents the nonlinear saturation function defined by

$$
\operatorname{sat}\left(u_{(i)}\right)= \begin{cases}u_{\max (i)} & \text { if } u_{(i)}>u_{\max (i)} ; \\ u_{(i)} & \text { if }-u_{\min (i)} \leq u_{(i)} \leq u_{\max (i)} \\ -u_{\min (i)} & \text { if } u_{(i)}<-u_{\min (i)}\end{cases}
$$

with $i \in\{1, \ldots, m\}$. Throughout this article, only symmetrical saturation functions are considered with

$$
u_{0(i)}=u_{\max (i)}=u_{\min (i)}, \forall i \in\{1, \ldots, m\} .
$$

\section{Event-triggered control subject to actuator saturation}

\subsection{Components of the event-triggered control loop}

Event-generator An event condition commonly used in the literature is given by deadband sampling (Otanez et al. 2002; Vasyutynskyy and Kabitzsch 2006), where a communication is invoked whenever the difference between the last transmitted information $\boldsymbol{y}\left(t_{k}\right)$ and the current measurement $\boldsymbol{y}(t)$ reaches an event threshold $\bar{e}$ :

$$
\left\|\boldsymbol{y}\left(t_{k}\right)-\boldsymbol{y}(t)\right\|=\bar{e} .
$$

Based on this event condition and by defining the output error according to

$$
\boldsymbol{e}(t)=\boldsymbol{y}\left(t_{k}\right)-\boldsymbol{y}(t)
$$


this section introduces a more general event condition which aims at keeping the output error $\boldsymbol{e}(t)$ in the set

$$
\mathcal{W}=\left\{\boldsymbol{e} \in \mathfrak{R}^{r}: \quad \boldsymbol{e}^{T} \boldsymbol{R} \boldsymbol{e} \leq \delta^{-1}\right\}
$$

with $\boldsymbol{R}=\boldsymbol{R}^{T} \succ 0$ and $\delta^{-1}>0$. This is obtained by invoking a communication, i.e., sending the current measurement $\boldsymbol{y}(t)$, whenever the output error reaches the boundary of the set $\mathcal{W}$ and, hence,

$$
\boldsymbol{e}(t) \in \partial \mathcal{W}=\left\{\boldsymbol{e} \in \mathfrak{R}^{r}: \boldsymbol{e}^{T} \boldsymbol{R} \boldsymbol{e}=\delta^{-1}\right\}
$$

holds with $\delta^{-1}$ denoting the event threshold and $t_{k}:=t$. Note that this condition corresponds to event condition 5 by setting $\boldsymbol{R}=\boldsymbol{I}_{r}$ and $\delta^{-1}=\bar{e}^{2}$.

Controller The plant 1, 2 is assumed to be controlled by a general linear dynamic output feedback controller which receives new information about the plant output $\boldsymbol{y}(t)$ only at event times $t_{k}$ with $k \in\{0,1,2, \ldots\}$. Hence, the event-triggered controller can be described during the time interval $\left[t_{k}, t_{k+1}\right)$ by

$$
\begin{aligned}
\dot{\boldsymbol{x}}_{c}(t) & =\tilde{\boldsymbol{A}}_{c} \boldsymbol{x}_{c}(t)+\tilde{\boldsymbol{B}}_{c} \boldsymbol{y}\left(t_{k}\right)+\tilde{\boldsymbol{B}}_{c W} \boldsymbol{w}(t), \quad \boldsymbol{x}_{c}\left(t_{k}\right)=\boldsymbol{x}_{c k} \\
\boldsymbol{u}(t) & =\tilde{\boldsymbol{C}}_{c} \boldsymbol{x}_{c}(t)+\tilde{\boldsymbol{D}}_{c} \boldsymbol{y}\left(t_{k}\right)+\tilde{\boldsymbol{D}}_{c W} \boldsymbol{w}(t)
\end{aligned}
$$

with $\boldsymbol{x}_{c} \in \mathfrak{R}^{n_{c}}$ the integrator state (controller state), where $\boldsymbol{x}_{c k}$ denotes the integrator state at time $t_{k}$, and $\boldsymbol{w} \in \mathfrak{R}^{s}$ represents reference signals to be followed. The matrices $\tilde{\boldsymbol{A}}_{c}, \tilde{\boldsymbol{B}}_{c}, \tilde{\boldsymbol{B}}_{c W}, \tilde{\boldsymbol{C}}_{c}, \tilde{\boldsymbol{D}}_{c}$ and $\tilde{\boldsymbol{D}}_{c W}$ denote real matrices of appropriate dimensions.

By replacing $\boldsymbol{y}\left(t_{k}\right)$ according to definition 6 the controller equations can be rewritten to get an equivalent continuous-time representation which holds for $t \geq 0$ :

$$
\begin{aligned}
\dot{\boldsymbol{x}}_{c}(t) & =\tilde{\boldsymbol{A}}_{c} \boldsymbol{x}_{c}(t)+\tilde{\boldsymbol{B}}_{c} \boldsymbol{y}(t)+\tilde{\boldsymbol{B}}_{c} \boldsymbol{e}(t)+\tilde{\boldsymbol{B}}_{c W} \boldsymbol{w}(t), \quad \boldsymbol{x}_{c}(0)=\boldsymbol{x}_{c 0} \\
\boldsymbol{u}(t) & =\tilde{\boldsymbol{C}}_{c} \boldsymbol{x}_{c}(t)+\tilde{\boldsymbol{D}}_{c} \boldsymbol{y}(t)+\tilde{\boldsymbol{D}}_{c} \boldsymbol{e}(t)+\tilde{\boldsymbol{D}}_{c W} \boldsymbol{w}(t) .
\end{aligned}
$$

In the following it is assumed that the controller parameters are designed in a way that the unconstrained continuous-time control loop 1, 2, 8, 9 with $\tilde{\boldsymbol{u}}(t)=\boldsymbol{u}(t)(\forall \boldsymbol{u})$ and $\boldsymbol{e}(t)=\mathbf{0}(\forall t)$ is stable.

\subsection{Description of the event-triggered control loop}

With plant 1, 2 and controller 8,9 and by introducing the augmented state vector $\boldsymbol{x}=\left[\begin{array}{ll}\boldsymbol{x}_{p} & \boldsymbol{x}_{c}\end{array}\right]^{T} \in \mathfrak{R}^{n_{p}+n_{c}}$, which includes both the plant state and the controller state, the event-triggered control loop can be described by the continuous-time state-space model

$$
\begin{aligned}
& \dot{\boldsymbol{x}}(t)=\boldsymbol{A x}(t)+\boldsymbol{B} \mathbf{s a t}(\boldsymbol{u}(t))+\boldsymbol{B}_{D} \boldsymbol{d}(t)+\boldsymbol{B}_{W} \boldsymbol{w}(t)+\boldsymbol{B}_{E} \boldsymbol{e}(t), \boldsymbol{x}(0)=\boldsymbol{x}_{0} \\
& \boldsymbol{u}(t)=\boldsymbol{K} \boldsymbol{x}(t)+\boldsymbol{K}_{W} \boldsymbol{w}(t)+\boldsymbol{K}_{E} \boldsymbol{e}(t) \\
& \boldsymbol{y}(t)=\boldsymbol{C} \boldsymbol{x}(t)
\end{aligned}
$$


with

$$
\begin{aligned}
& \boldsymbol{A}=\left[\begin{array}{cc}
\tilde{\boldsymbol{A}} & \boldsymbol{O} \\
\tilde{\boldsymbol{B}}_{c} \tilde{\boldsymbol{C}} & \tilde{\boldsymbol{A}}_{c}
\end{array}\right], \quad \boldsymbol{B}=\left[\begin{array}{c}
\tilde{\boldsymbol{B}} \\
\boldsymbol{O}
\end{array}\right], \quad \boldsymbol{B}_{D}=\left[\begin{array}{c}
\tilde{\boldsymbol{B}}_{D} \\
\boldsymbol{O}
\end{array}\right], \quad \boldsymbol{B}_{W}=\left[\begin{array}{c}
\boldsymbol{O} \\
\tilde{\boldsymbol{B}}_{c W}
\end{array}\right], \quad \boldsymbol{B}_{E}=\left[\begin{array}{c}
\boldsymbol{O} \\
\tilde{\boldsymbol{B}}_{c}
\end{array}\right] \\
& \boldsymbol{K}=\left[\begin{array}{lll}
\tilde{\boldsymbol{D}}_{c} \tilde{\boldsymbol{C}} & \tilde{\boldsymbol{C}}_{c}
\end{array}\right], \quad \boldsymbol{K}_{W}=\tilde{\boldsymbol{D}}_{c W}, \quad \boldsymbol{K}_{E}=\tilde{\boldsymbol{D}}_{c}, \quad \boldsymbol{C}=\left[\begin{array}{ll}
\tilde{\boldsymbol{C}} \boldsymbol{O}
\end{array}\right] .
\end{aligned}
$$

Equations 10-12 show that both the plant and the controller are affected by the output error signal $\boldsymbol{e}(t)$ introduced by the event-triggered sampling. Moreover, since the controller dynamics are included in the plant dynamics the controller can be represented as a pure proportional controller. This is illustrated in Fig. 2.

Dead-zone nonlinearity Henceforth, the saturation nonlinearity is replaced by a decentralized dead-zone nonlinearity $\phi(t)$ according to

$$
\boldsymbol{\phi}(\boldsymbol{u}(t))=\operatorname{sat}(\boldsymbol{u}(t))-\boldsymbol{u}(t) .
$$

The main benefit of this transformation is that it allows using a modified sector condition. This condition specifically applies to dead-zone nonlinearities potentially resulting in less conservative results compared to using classical sector conditions (Tarbouriech et al. 2006).

Lemma 1 (Tarbouriech et al. 2006) If $\boldsymbol{v} \in \mathfrak{R}^{m}$ and $z \in \mathfrak{R}^{m}$ are elements of the set

$$
\mathcal{S}=\left\{\boldsymbol{v} \in \mathfrak{R}^{m}, \quad z \in \mathfrak{R}^{m}:\left|v_{(i)}-z_{(i)}\right| \leq u_{0(i)}, \quad \forall i \in\{1, \ldots, m\}\right\}
$$

then the nonlinearity $\phi(v)$ satisfies the inequality

$$
\boldsymbol{\phi}(\boldsymbol{v})^{T} \boldsymbol{T}(\boldsymbol{\phi}(\boldsymbol{v})+\boldsymbol{z}) \leq 0
$$

for any diagonal positive definite matrix $\boldsymbol{T} \in \mathfrak{R}^{m \times m}$.

By using transformation 13, the state-space model 10, 11 can be rewritten as

$$
\begin{aligned}
\dot{\boldsymbol{x}}(t)= & \overline{\boldsymbol{A}} \boldsymbol{x}(t)+\overline{\boldsymbol{B}} \boldsymbol{\phi}\left(\boldsymbol{K} \boldsymbol{x}(t)+\boldsymbol{K}_{W} \boldsymbol{w}(t)+\boldsymbol{K}_{E} \boldsymbol{e}(t)\right) \\
& +\boldsymbol{B}_{D} \boldsymbol{d}(t)+\left(\boldsymbol{B}_{W}+\boldsymbol{B} \boldsymbol{K}_{W}\right) \boldsymbol{w}(t)+\left(\boldsymbol{B}_{E}+\boldsymbol{B} \boldsymbol{K}_{E}\right) \boldsymbol{e}(t), \boldsymbol{x}(0)=\boldsymbol{x}_{0} \\
\boldsymbol{y}(t)= & \boldsymbol{C} \boldsymbol{x}(t)
\end{aligned}
$$

with

$$
\overline{\boldsymbol{A}}=\boldsymbol{A}+\boldsymbol{B} \boldsymbol{K}, \quad \overline{\boldsymbol{B}}=\boldsymbol{B} .
$$

Fig. 2 Continuous-time representation of the event-triggered control loop with augmented plant

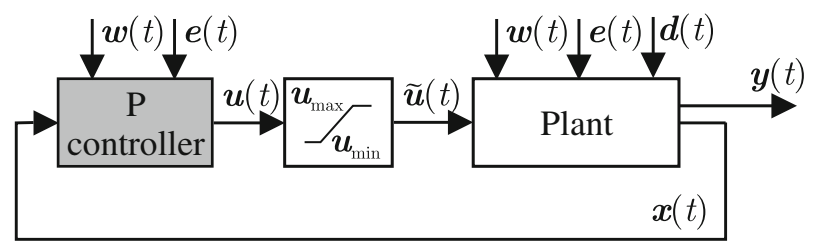


Note that the matrix $\overline{\boldsymbol{A}}$ is Hurwitz due to assuming that the controller 8, 9 leads to a stable unconstrained continuous-time control loop. The model 14,15 is used for the stability analysis carried out next.

\subsection{Stability analysis}

For the sake of simplicity, the disturbance and reference signals are firstly set to zero, i.e.,

$$
\boldsymbol{d}(t)=\boldsymbol{w}(t)=\mathbf{0}, \forall t
$$

Theorem 1 Suppose $\boldsymbol{d}(t)=\boldsymbol{w}(t)=\mathbf{0}, \forall t$. If there exist a symmetric positive definite matrix $\boldsymbol{W} \in \mathfrak{R}^{n \times n}$, a positive definite diagonal matrix $\boldsymbol{S} \in \mathfrak{R}^{m \times m}$, a matrix $\boldsymbol{Z} \in \mathfrak{R}^{m \times n}$, a positive scalar $\eta$ and two a priori fixed positive scalars $\tau_{1}$ and $\tau_{2}$ satisfying

$$
\begin{aligned}
& {\left[\begin{array}{ccc}
\boldsymbol{W} \overline{\boldsymbol{A}}^{T}+\overline{\boldsymbol{A}} \boldsymbol{W}+\tau_{1} \boldsymbol{W} & \star & \star \\
\boldsymbol{S} \overline{\boldsymbol{B}}^{T}-\boldsymbol{K} \boldsymbol{W}-\boldsymbol{Z} & -2 \boldsymbol{S} & \star \\
\left(\boldsymbol{B}_{E}+\boldsymbol{B} \boldsymbol{K}_{E}\right)^{T} & -\boldsymbol{K}_{E}^{T}-\tau_{2} \boldsymbol{R}
\end{array}\right] \prec 0} \\
& -\tau_{1} \delta+\tau_{2} \eta<0 \\
& {\left[\begin{array}{cc}
\boldsymbol{W} & \boldsymbol{Z}_{(i)}^{T} \\
\boldsymbol{Z}_{(i)} & \eta u_{0(i)}^{2}
\end{array}\right] \succeq 0, \quad i \in\{1, \ldots, m\}}
\end{aligned}
$$

then for any $\boldsymbol{e} \in \mathcal{W}=\left\{\boldsymbol{e} \in \mathfrak{R}^{r}: \boldsymbol{e}^{T} \boldsymbol{R} \boldsymbol{e} \leq \delta^{-1}\right\}$ and $\boldsymbol{x}_{0} \in \mathcal{E}(\boldsymbol{P}, \eta)$ with

$$
\mathcal{E}(\boldsymbol{P}, \eta)=\left\{\boldsymbol{x} \in \mathfrak{R}^{n_{p}+n_{c}}: \boldsymbol{x}^{T} \boldsymbol{P} \boldsymbol{x} \leq \eta^{-1}\right\}
$$

and $\boldsymbol{P}=\boldsymbol{W}^{-1}$, the state $\boldsymbol{x}(t)$ of the event-triggered control loop 14, 15 does not leave the ellipsoid $\mathcal{E}(\boldsymbol{P}, \eta)$ for all future times $t>0$.

Proof The proof follows the procedure introduced in Tarbouriech et al. (2011).

By setting $\boldsymbol{v}=\boldsymbol{u}=\boldsymbol{K} \boldsymbol{x}+\boldsymbol{K}_{E} \boldsymbol{e}$ and $\boldsymbol{z}=\boldsymbol{u}+\boldsymbol{G} \boldsymbol{x}=\boldsymbol{K} \boldsymbol{x}+\boldsymbol{K}_{E} \boldsymbol{e}+\boldsymbol{G} \boldsymbol{x}$, Lemma 1 guarantees that any $\boldsymbol{x}$ belonging to the set

$$
\mathcal{S}_{\boldsymbol{G}}=\left\{\boldsymbol{x} \in \mathfrak{R}^{n_{p}+n_{c}}: \quad\left|\boldsymbol{G}_{(i)} \boldsymbol{x}\right| \leq u_{0(i)} \quad \forall i \in\{1, \ldots, m\}\right\}
$$

satisfies the sector condition

$$
\boldsymbol{\phi}(\boldsymbol{u})^{T} \boldsymbol{T}(\boldsymbol{\phi}(\boldsymbol{u})+\boldsymbol{u}+\boldsymbol{G} \boldsymbol{x}) \leq 0 .
$$

Consider the quadratic Lyapunov function candidate

$$
V=\boldsymbol{x}^{T} \boldsymbol{P} \boldsymbol{x}
$$


with $\boldsymbol{P}=\boldsymbol{P}^{T} \succ 0$, which defines the ellipsoid $\mathcal{E}(\boldsymbol{P}, \eta)$ according to Eq. 19. This ellipsoid is included in the set $\mathcal{S}_{\boldsymbol{G}}$ if the inequalities

$$
\begin{aligned}
{\left[\begin{array}{cc}
\boldsymbol{P} & \boldsymbol{G}_{(i)}^{T} \\
\boldsymbol{G}_{(i)} & \eta u_{0(i)}^{(2)}
\end{array}\right] } & \succeq 0 \text { for } i=1, \ldots, m \\
\boldsymbol{P} & \succeq 0
\end{aligned}
$$

hold (see Tarbouriech et al. 2011 for a detailed proof of this fact). Then, it follows that sector condition 20 is satisfied for every $\boldsymbol{x} \in \mathcal{E}(\boldsymbol{P}, \eta)$. By setting $\boldsymbol{W}=\boldsymbol{P}^{-1}$ and $\boldsymbol{Z}=\boldsymbol{G} \boldsymbol{W}$, inequality 22 can be rewritten to get Eq. 18 . Inequality 23 is automatically fulfilled as a requirement for Eq. 21.

Next, conditions are derived to prove that the time derivative $\dot{V}(\boldsymbol{x})$ satisfies $\dot{V}(\boldsymbol{x})<0$ for any

$$
\boldsymbol{x} \notin \operatorname{int} \mathcal{E}(\boldsymbol{P}, \eta)=\left\{\boldsymbol{x} \in \mathfrak{R}^{n_{p}+n_{c}}: \boldsymbol{x}^{T} \boldsymbol{P} \boldsymbol{x}<\eta^{-1}\right\}
$$

and any $\boldsymbol{e} \in \mathcal{W}$. By applying the S-procedure, the condition

$$
\dot{V}(\boldsymbol{x})+\tau_{1}\left(\boldsymbol{x}^{T} \boldsymbol{P} \boldsymbol{x}-\eta^{-1}\right)+\tau_{2}\left(\delta^{-1}-\boldsymbol{e}^{T} \boldsymbol{R} \boldsymbol{e}\right)<0
$$

with $\tau_{1}, \tau_{2}>0$ is obtained. For further analysis, Eq. 24 is split into two inequalities:

$$
\begin{array}{r}
-\tau_{1} \delta+\tau_{2} \eta<0 \\
\dot{V}(\boldsymbol{x})+\tau_{1} \boldsymbol{x}^{T} \boldsymbol{P} \boldsymbol{x}-\tau_{2} \boldsymbol{e}^{T} \boldsymbol{R} \boldsymbol{e}<0 .
\end{array}
$$

Equation 25 directly results in condition 17 . Equation 26 can be extended by using the sector condition 20 for $\boldsymbol{x} \in \partial \mathcal{E}(\boldsymbol{P}, \eta)$ and using the fact that

$$
\begin{aligned}
\dot{V}(\boldsymbol{x}) & +\tau_{1} \boldsymbol{x}^{T} \boldsymbol{P} \boldsymbol{x}-\tau_{2} \boldsymbol{e}^{T} \boldsymbol{R} \boldsymbol{e} \\
\leq & \dot{V}(\boldsymbol{x})+\tau_{1} \boldsymbol{x}^{T} \boldsymbol{P} \boldsymbol{x}-\tau_{2} \boldsymbol{e}^{T} \boldsymbol{R} \boldsymbol{e}-2 \boldsymbol{\phi}^{T} \boldsymbol{T}\left(\boldsymbol{\phi}+\boldsymbol{K} \boldsymbol{x}+\boldsymbol{K}_{E} \boldsymbol{e}+\boldsymbol{G} \boldsymbol{x}\right)<0
\end{aligned}
$$

holds. By using the system representation 14 and by transforming the decision variables according to $\boldsymbol{W}=\boldsymbol{P}^{-1}, \boldsymbol{S}=\boldsymbol{T}^{-1}$ and $\boldsymbol{Z}=\boldsymbol{G} \boldsymbol{W}$, finally condition 16 is obtained which concludes the proof.

The result shows that for any state $\boldsymbol{x}\left(t_{1}\right)$ located on the boundary of ellipsoid 19, i.e., $\boldsymbol{x}\left(t_{1}\right) \in \partial \mathcal{E}(\boldsymbol{P}, \eta)$, and $\boldsymbol{e}(t) \in \mathcal{W}$, it follows that $\dot{V}\left(\boldsymbol{x}\left(t_{1}\right)\right)<0$. Thus, $\boldsymbol{x}\left(t_{1}+\Delta t\right)$ will lie in the interior of the ellipsoid for any arbitrary small time step $\Delta t$. Consequently, the ellipsoid $\mathcal{E}(\boldsymbol{P}, \eta)$ is a positive invariant set for the event-triggered control loop 14, 15 (see Blanchini 1999).

If $\boldsymbol{e}(t)=\mathbf{0}$ holds for all times $t \geq 0$, Eq. 26 turns into

$$
\dot{V}(\boldsymbol{x})<-\tau_{1} \boldsymbol{x}^{T} \boldsymbol{P} \boldsymbol{x}<0 .
$$

This inequality ensures that $\dot{V}(\boldsymbol{x})<0$ holds for all $\boldsymbol{x} \in \mathcal{E}(\boldsymbol{P}, \eta)$. Hence, $\mathcal{E}(\boldsymbol{P}, \eta)$ describes a region of asymptotic stability for system 14,15 . As $\boldsymbol{e}(t)=\mathbf{0}(\forall t)$ corresponds to a continuous-time information exchange which should be generally avoided when considering event-triggered control, this fact is neglected in the following. 
The LMI conditions introduced by Theorem 1 can be used to check whether or not a stability region exists for the event-triggered control loop subject to a predefined event threshold and zero exogenous signals. Moreover, Theorem 1 can be also used to derive the maximum stability region $\mathcal{E}(\boldsymbol{P}, \eta)$ for a fixed event threshold $\delta^{-1}$ or to find the maximum event threshold leading to a feasible set of inequalities. Suitable optimization methods are discussed in Section 5.

\subsection{Minimum inter-event time}

The minimum inter-event time between two consecutive events is given by

$$
T_{\min }=\min _{k}\left\{t_{k+1}-t_{k}\right\}, \quad k=0,1,2, \ldots .
$$

The following theorem shows that there exists a lower bound on the minimum interevent time and, hence, Zeno behavior can be excluded.

Theorem 2 Assume that the event-triggered control loop 14, 15 with $\boldsymbol{w}(t)=\boldsymbol{d}(t)=$ $\mathbf{0}, \forall t$, satisfies the inequalities 16-18, then for $\boldsymbol{x}_{0} \in \mathcal{E}(\boldsymbol{P}, \eta)$ the minimum inter-event time $T_{\min }$ is lower bounded by

$$
T_{\min } \geq \bar{T}=\arg \min _{t}\left\{\tilde{e}(t)=\sqrt{\frac{1}{\delta\|\boldsymbol{R}\|}}\right\}
$$

with

$$
\tilde{e}(t)=\max _{t}\left\|\tilde{\boldsymbol{C}}\left(\mathrm{e}^{\tilde{\boldsymbol{A}} t}-\boldsymbol{I}_{n}\right)\right\| x_{\max }+\int_{0}^{t}\left\|\tilde{\boldsymbol{C}} \mathrm{e}^{\tilde{\boldsymbol{A}}(t-\tau)} \boldsymbol{B}\right\| \mathrm{d} \tau u_{0 \max }
$$

and

$$
\begin{aligned}
x_{\max } & =\max _{\boldsymbol{x} \in \mathcal{E}(\boldsymbol{P}, \eta)}\|\boldsymbol{x}\| \\
u_{0 \max } & =\max _{i \in\{1,2, \ldots, m\}} u_{0(i)} .
\end{aligned}
$$

Proof The plant dynamics are described by the state-space model 1, 2. Considering $\boldsymbol{d}(t)=\mathbf{0}$, the output trajectory is given by

$$
\boldsymbol{y}(t)=\tilde{\boldsymbol{C}} \mathrm{e}^{\tilde{\boldsymbol{A}} t} \boldsymbol{x}_{0}+\tilde{\boldsymbol{C}} \int_{0}^{t} \mathrm{e}^{\tilde{\boldsymbol{A}}(t-\tau)} \boldsymbol{B} \tilde{\boldsymbol{u}}(\tau) \mathrm{d} \tau .
$$

The norm of the output error

$$
\|\boldsymbol{e}(t)\|=\left\|\boldsymbol{y}\left(t_{k}\right)-\boldsymbol{y}(t)\right\|
$$

introduced by considering the over-approximation

$$
\boldsymbol{e}^{T} \boldsymbol{R} \boldsymbol{e} \leq\|\boldsymbol{e}\|^{2}\|\boldsymbol{R}\|
$$

can be upper bounded by

$$
\|\boldsymbol{e}(t)\|=\left\|\tilde{\boldsymbol{C}}\left(\mathrm{e}^{\tilde{\boldsymbol{A}} t}-\boldsymbol{I}_{n}\right) \boldsymbol{x}_{0}+\tilde{\boldsymbol{C}} \int_{0}^{t} \mathrm{e}^{\tilde{\boldsymbol{A}}(t-\tau)} \boldsymbol{B} \tilde{\boldsymbol{u}}(\tau) \mathrm{d} \tau\right\| \leq \tilde{e}(t)
$$


$\left(t_{k}=0\right)$ with $\tilde{e}(t)$ given by Eq. 28 . As $\|\boldsymbol{u}\| \leq u_{0 \max }$ holds independent of the control signal produced by controller due to the actuator limitations and since an event is generated whenever the equation $\boldsymbol{e}^{T} \boldsymbol{R} \boldsymbol{e}=\delta^{-1}$ holds, $\tilde{e}(t)$ can be used to derive a lower bound on the minimum inter-event time by means of relation 27 .

The theorem shows that the information exchange over the feedback link can be arbitrarily adapted by choosing $\delta$ accordingly. However, as this parameter likewise affects the stability conditions according to Eq. 17, a small $\delta$ might lead to an infeasible inequality set Eqs. 16-18.

\subsection{Nonzero exogenous signals}

In practical applications, disturbances and reference signals cannot be neglected. Therefore, $\boldsymbol{d}(t) \neq \mathbf{0}$ and $\boldsymbol{w}(t) \neq \mathbf{0}$ need to be generally considered within the stability analysis of the event-triggered control loop 14, 15.

However, in order to derive stability conditions based on the previous method, disturbances $\boldsymbol{d}(t)$ as well as reference signals $\boldsymbol{w}(t)$ have to be bounded. In the following, is assumed that both signals are bounded by a quadratic norm, where $\boldsymbol{d}(t)$ belongs to the set $\mathcal{V}_{D}$ according to Eq. 3 and $w(t)$ belongs to the set

$$
\mathcal{V}_{W}=\left\{\boldsymbol{w} \in \mathfrak{R}^{s}: \boldsymbol{w}^{T} \boldsymbol{Q}_{W} \boldsymbol{w} \leq \epsilon_{W}^{-1}\right\}, \quad \boldsymbol{Q}_{W}=\boldsymbol{Q}_{W}^{T} \succ 0, \epsilon_{W}>0 .
$$

Taking these exogenous signals into account Theorem 2 needs to be modified to get the following result.

Corollary 1 If there exist a symmetric positive definite matrix $\boldsymbol{W} \in \Re^{n \times n}$, a positive definite diagonal matrix $\boldsymbol{S} \in \mathfrak{R}^{m \times m}$, a matrix $\boldsymbol{Z} \in \mathfrak{R}^{m \times n}$, a positive scalar $\eta$ and four a priori fixed positive scalars $\tau_{1}, \tau_{2}, \tau_{3}$ and $\tau_{4}$ satisfying

$$
\begin{gathered}
{\left[\begin{array}{ccccc}
\boldsymbol{W} \overline{\boldsymbol{A}}^{T}+\overline{\boldsymbol{A}} \boldsymbol{W}+\tau_{1} \boldsymbol{W} & \star & \star & \star & \star \\
\boldsymbol{S} \overline{\boldsymbol{B}}^{T}-\boldsymbol{K} \boldsymbol{W}-\boldsymbol{Z} & -2 \boldsymbol{S} & \star & \star & \star \\
\left(\boldsymbol{B}_{E}+\boldsymbol{B} \boldsymbol{K}_{E}\right)^{T} & -\boldsymbol{K}_{E}^{T} & -\tau_{2} \boldsymbol{R} & \star & \star \\
\boldsymbol{B}_{D}^{T} & \mathbf{0} & \mathbf{0} & -\tau_{3} \boldsymbol{Q}_{D} & \star \\
\left(\boldsymbol{B}_{W}+\boldsymbol{B} \boldsymbol{K}_{W}\right)^{T} & \boldsymbol{K}_{W}^{T} & \mathbf{0} & \mathbf{0} & -\tau_{4} \boldsymbol{Q}_{W}
\end{array}\right] \prec 0} \\
-\tau_{1} \delta \epsilon_{D} \epsilon_{W}+\tau_{2} \eta \epsilon_{D} \epsilon_{W}+\tau_{3} \eta \delta \epsilon_{W}+\tau_{4} \eta \delta \epsilon_{D}<0 \\
{\left[\begin{array}{cc}
\boldsymbol{W} & \boldsymbol{Z}_{(i)}^{T} \\
\boldsymbol{Z}_{(i)} & \eta u_{0(i)}^{2}
\end{array}\right] \succeq 0, \quad i \in\{1, \ldots, m\}}
\end{gathered}
$$

then for any $\boldsymbol{e} \in \mathcal{W}, \boldsymbol{d} \in \mathcal{V}_{D}, \boldsymbol{w} \in \mathcal{V}_{W}$ and $\boldsymbol{x}_{0} \in \mathcal{E}(\boldsymbol{P}, \eta)$ with $\boldsymbol{P}=\boldsymbol{W}^{-1}$, the state $\boldsymbol{x}(t)$ of the event-triggered control loop 14,15 does not leave the ellipsoid $\mathcal{E}(\boldsymbol{P}, \eta)$ for all future times $t>0$. 
Proof In addition to the requirements of Theorem 1, Eqs. 3 and 31 need to be considered. Therefore, Eq. 24 can be extended by

$$
\begin{aligned}
& \dot{V}(\boldsymbol{x})+\tau_{1}\left(\boldsymbol{x}^{T} \boldsymbol{P} \boldsymbol{x}-\eta^{-1}\right)+\tau_{2}\left(\delta^{-1}-\boldsymbol{e}^{T} \boldsymbol{R} \boldsymbol{e}\right) \\
& \quad+\tau_{3}\left(\epsilon_{D}^{-1}-\boldsymbol{d}^{T} \boldsymbol{Q}_{D} \boldsymbol{d}\right)+\tau_{4}\left(\epsilon_{W}^{-1}-\boldsymbol{w}^{T} \boldsymbol{Q}_{W} \boldsymbol{w}\right)<0
\end{aligned}
$$

with $\tau_{1}, \tau_{2}, \tau_{3}, \tau_{4}>0$. Again, this inequality can be split into two inequalities:

$$
\begin{array}{r}
-\tau_{1} \delta \epsilon_{D} \epsilon_{W}+\tau_{2} \eta \epsilon_{D} \epsilon_{W}+\tau_{3} \eta \delta \epsilon_{W}+\tau_{4} \eta \delta \epsilon_{D}<0 \\
\dot{V}(\boldsymbol{x})+\tau_{1} \boldsymbol{x}^{T} \boldsymbol{P} \boldsymbol{x}-\tau_{2} \boldsymbol{e}^{T} \boldsymbol{R} \boldsymbol{e}-\tau_{3} \boldsymbol{d}^{T} \boldsymbol{Q}_{D} \boldsymbol{d}-\tau_{4} \boldsymbol{w}^{T} \boldsymbol{Q}_{W} \boldsymbol{w}<0 .
\end{array}
$$

Equation 35 directly leads to condition 33. Extending Eq. 36 by using sector condition 20 with $\boldsymbol{u}=\boldsymbol{K} \boldsymbol{x}+\boldsymbol{K}_{W} \boldsymbol{w}+\boldsymbol{K}_{E} \boldsymbol{e}$ yields

$$
\begin{aligned}
\dot{V}(\boldsymbol{x}) & +\tau_{1} \boldsymbol{x}^{T} \boldsymbol{P} \boldsymbol{x}-\tau_{2} \boldsymbol{e}^{T} \boldsymbol{R} \boldsymbol{e}-\tau_{3} \boldsymbol{d}^{T} \boldsymbol{Q}_{D} \boldsymbol{d}-\tau_{4} \boldsymbol{w}^{T} \boldsymbol{Q}_{W} \boldsymbol{w} \\
\leq & \dot{V}(\boldsymbol{x})+\tau_{1} \boldsymbol{x}^{T} \boldsymbol{P} \boldsymbol{x}-\tau_{2} \boldsymbol{e}^{T} \boldsymbol{R} \boldsymbol{e}-\tau_{3} \boldsymbol{d}^{T} \boldsymbol{Q}_{D} \boldsymbol{d}-\tau_{4} \boldsymbol{w}^{T} \boldsymbol{Q}_{W} \boldsymbol{w} \\
& -2 \boldsymbol{\phi}^{T} \boldsymbol{T}\left(\boldsymbol{\phi}+\boldsymbol{K} \boldsymbol{x}+\boldsymbol{K}_{W} \boldsymbol{w}+\boldsymbol{K}_{E} \boldsymbol{e}+\boldsymbol{G} \boldsymbol{x}\right)<0 .
\end{aligned}
$$

Finally, by considering Eq. 14 and by using the transformations $\boldsymbol{W}=\boldsymbol{P}^{-1}, \boldsymbol{S}=\boldsymbol{T}^{-1}$ and $\boldsymbol{Z}=\boldsymbol{G} \boldsymbol{W}$, condition 32 is obtained.

Moreover, the next result brings about how the lower bound on the minimum inter-event time is affected by exogenous signals.

Corollary 2 Assume that the event-triggered control loop 14, 15 with $\boldsymbol{w}(t)$ and $\boldsymbol{d}(t)$ according to Eqs. 3 and 31 satisfies the inequalities 32-34, then for $\boldsymbol{x}_{0} \in \mathcal{E}(\boldsymbol{P}, \eta)$ the minimum inter-event time $T_{\min }$ is lower bounded by

$$
T_{\min } \geq \bar{T}_{d}=\arg \min _{t}\left\{\tilde{e}_{d}(t)=\sqrt{\frac{1}{\delta\|\boldsymbol{R}\|}}\right\}
$$

with

$$
\tilde{e}_{d}(t)=\max _{t}\left\|\tilde{\boldsymbol{C}}\left(\mathrm{e}^{\tilde{\boldsymbol{A}} t}-\boldsymbol{I}_{n}\right)\right\| x_{\max }+\int_{0}^{t}\left\|\tilde{\boldsymbol{C}} \mathrm{e}^{\tilde{\boldsymbol{A}}(t-\tau)}\right\| \mathrm{d} \tau\left(\|\tilde{\boldsymbol{B}}\| u_{0 \max }+\left\|\tilde{\boldsymbol{B}}_{D}\right\| d_{\max }\right),
$$

$x_{\max }$ and $u_{0 \max }$ according to Eqs. 29 and 30, and

$$
d_{\max }=\max _{\boldsymbol{d} \in \mathcal{V}_{D}}\|\boldsymbol{d}\|
$$

Proof The plant dynamics are described by the state-space model 1,2 which yields the output trajectory

$$
\boldsymbol{y}(t)=\tilde{\boldsymbol{C}} \mathrm{e}^{\tilde{\boldsymbol{A}} t} \boldsymbol{x}_{0}+\tilde{\boldsymbol{C}} \int_{0}^{t} \mathrm{e}^{\tilde{\boldsymbol{A}}(t-\tau)}\left(\tilde{\boldsymbol{B}} \tilde{\boldsymbol{u}}(\tau)+\tilde{\boldsymbol{B}}_{D} \boldsymbol{d}(\tau)\right) \mathrm{d} \tau .
$$


By considering the same over-approximation as in the proof of Theorem 2, the norm of the output error can be upper bounded by

$$
\|\boldsymbol{e}(t)\|=\left\|\tilde{\boldsymbol{C}}\left(\mathrm{e}^{\tilde{\boldsymbol{A}} t}-\boldsymbol{I}_{n}\right) \boldsymbol{x}_{0}+\tilde{\boldsymbol{C}} \int_{0}^{t} \mathrm{e}^{\tilde{\boldsymbol{A}}(t-\tau)}\left(\tilde{\boldsymbol{B}} \tilde{\boldsymbol{u}}(\tau)+\tilde{\boldsymbol{B}}_{D} \boldsymbol{d}(\tau)\right) \mathrm{d} \tau\right\| \leq \tilde{e}_{d}(t)
$$

$\left(t_{k}=0\right)$ with $\tilde{e}_{d}(t)$ given by Eq. 38. By means of relation $37, \tilde{e}_{d}(t)$ is used to derive a lower bound on the minimum inter-event time.

Note that the communication bound is not affected by the reference signal $\boldsymbol{w}(t)$. The reason for this is given by the fact that $\boldsymbol{w}(t)$ only directly affects the output of the controller (Eq. 9), which is upper bounded by the saturation limits, and the controller state $\boldsymbol{x}_{c}(t)$ (Eq. 8). However, the controller state is not used for the event generation.

\section{Anti-windup compensation}

\subsection{Event-triggered control loop with anti-windup compensation}

For continuous-time controlled systems, there exists a large repertoire of so-called anti-windup techniques to compensate the potential performance degradation due to saturating actuators (see Tarbouriech et al. 2011; Zaccarian and Teel 2011). This section presents an extensions of the previous event-triggered control loop by using a static anti-windup mechanism which is illustrated in Fig. 3 for event-triggered PI control, i.e., $\tilde{\boldsymbol{A}}_{c}=\boldsymbol{O}, \tilde{\boldsymbol{B}}_{c}=\boldsymbol{I}_{r}, \tilde{\boldsymbol{B}}_{c W}=-\boldsymbol{I}_{r}, \tilde{\boldsymbol{C}}_{c}=\boldsymbol{K}_{I}, \tilde{\boldsymbol{D}}_{c}=\boldsymbol{K}_{P}, \tilde{\boldsymbol{D}}_{c W}=-\boldsymbol{K}_{P}(\mathrm{cf}$. Eqs. 8 and 9).

The main characteristic of this scheme is that the difference between the actual actuator output and the unconstraint controller output, which corresponds to the dead-zone nonlinearity 13 according to sat $(\boldsymbol{u}(t))-\boldsymbol{u}(t)=\boldsymbol{\phi}(\boldsymbol{u}(t))$, is fed back through the static gain $\boldsymbol{K}_{A W}$ in order to affect the evolution of the controller state $\boldsymbol{x}_{c}(t)$. Thereby, the controller Eq. 8 has to be extended in the following way:

$$
\dot{\boldsymbol{x}}_{c}(t)=\tilde{\boldsymbol{A}}_{c} \boldsymbol{x}_{c}(t)+\tilde{\boldsymbol{B}}_{c} \boldsymbol{y}(t)+\tilde{\boldsymbol{B}}_{c} \boldsymbol{e}(t)+\tilde{\boldsymbol{B}}_{c W} \boldsymbol{w}(t)+\boldsymbol{K}_{A W} \boldsymbol{\phi}(\boldsymbol{u}(t)), \quad \boldsymbol{x}_{c}(0)=\boldsymbol{x}_{c 0} .
$$

Fig. 3 Event-triggered PI controller with static anti-windup compensation

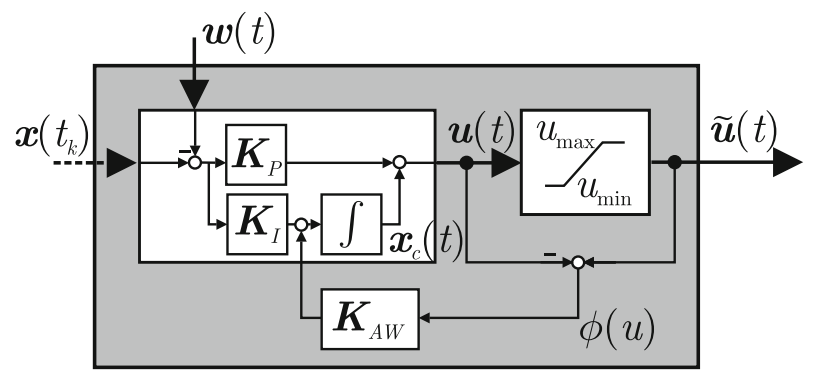


By using the plant description 1, 2, the adapted controller state 39, the controller output 9 and the dead-zone transformation 13, the closed-loop state-space representation reads

$$
\begin{aligned}
\dot{\boldsymbol{x}}(t)= & \overline{\boldsymbol{A}} \boldsymbol{x}(t)+\overline{\boldsymbol{B}}_{A W} \boldsymbol{\phi}\left(\boldsymbol{K} \boldsymbol{x}(t)+\boldsymbol{K}_{W} \boldsymbol{w}(t) \boldsymbol{K}_{E} \boldsymbol{e}(t)\right) \\
& +\boldsymbol{B}_{D} \boldsymbol{d}(t)+\left(\boldsymbol{B}_{W}+\boldsymbol{B} \boldsymbol{K}_{W}\right) \boldsymbol{w}(t)+\left(\boldsymbol{B}_{E}+\boldsymbol{B} \boldsymbol{K}_{E}\right) \boldsymbol{e}(t), \quad \boldsymbol{x}(0)=\boldsymbol{x}_{0} \\
\boldsymbol{y}(t)= & \boldsymbol{C} \boldsymbol{x}(t)
\end{aligned}
$$

with

$$
\overline{\boldsymbol{B}}_{A W}=\left[\begin{array}{c}
\tilde{\boldsymbol{B}} \\
\boldsymbol{K}_{A W}
\end{array}\right]
$$

The difference to model 14 is given by the different definition of the matrix $\overline{\boldsymbol{B}}$. Therefore, by adapting the matrix $\overline{\boldsymbol{B}}$ to $\overline{\boldsymbol{B}}_{A W}$, the method presented in Section 3 can be used straightforward to calculate the regions of stability for event-triggered control with anti-windup compensation.

\subsection{Stability analysis}

Corollary 3 If there exist a symmetric positive definite matrix $\boldsymbol{W} \in \mathfrak{R}^{n \times n}$, a positive definite diagonal matrix $\boldsymbol{S} \in \mathfrak{R}^{m \times m}$, a matrix $\boldsymbol{Z} \in \mathfrak{R}^{m \times n}$, a positive scalar $\eta$ and four a priori fixed positive scalars $\tau_{1}, \tau_{2}, \tau_{3}$ and $\tau_{4}$ satisfying

$$
\begin{gathered}
{\left[\begin{array}{ccccc}
\boldsymbol{W} \overline{\boldsymbol{A}}^{T}+\overline{\boldsymbol{A}} \boldsymbol{W}+\tau_{1} \boldsymbol{W} & \star & \star & \star & \star \\
\boldsymbol{S} \overline{\boldsymbol{B}}_{A W}^{T}-\boldsymbol{K} \boldsymbol{W}-\boldsymbol{Z} & -2 \boldsymbol{S} & \star & \star & \star \\
\left(\boldsymbol{B}_{E}+\boldsymbol{B} \boldsymbol{K}_{E}\right)^{T} & -\boldsymbol{K}_{E}^{T} & -\tau_{2} \boldsymbol{R} & \star & \star \\
\boldsymbol{B}_{D}^{T} & \mathbf{0} & \mathbf{0} & -\tau_{3} \boldsymbol{Q}_{D} & \star \\
\left(\boldsymbol{B}_{W}+\boldsymbol{B} \boldsymbol{K}_{W}\right)^{T} & \boldsymbol{K}_{W}^{T} & \mathbf{0} & \mathbf{0} & -\tau_{4} \boldsymbol{Q}_{W}
\end{array}\right] \prec 0} \\
-\tau_{1} \delta \epsilon_{D} \epsilon_{W}+\tau_{2} \eta \epsilon_{D} \epsilon_{W}+\tau_{3} \eta \delta \epsilon_{W}+\tau_{4} \eta \delta \epsilon_{D}<0 \\
{\left[\begin{array}{cc}
\boldsymbol{W} & \boldsymbol{Z}_{(i)}^{T} \\
\boldsymbol{Z}_{(i)} & \eta u_{0(i)}^{2}
\end{array}\right] \succeq 0, \quad i \in\{1, \ldots, m\}}
\end{gathered}
$$

then for any $\boldsymbol{e} \in \mathcal{W}, \boldsymbol{d} \in \mathcal{V}_{D}, \boldsymbol{w} \in \mathcal{V}_{W}$ and $\boldsymbol{x}_{0} \in \mathcal{E}(\boldsymbol{P}, \eta)$ with $\boldsymbol{P}=\boldsymbol{W}^{-1}$, the state $\boldsymbol{x}(t)$ of the event-triggered control loop 40-42 does not leave the ellipsoid $\mathcal{E}(\boldsymbol{P}, \eta)$ for all future times $t>0$.

The only difference to the previous result (Corollary 1) is that the matrix $\overline{\boldsymbol{B}}_{A W}$ now includes the anti-windup gain $\boldsymbol{K}_{A W}$. This parameter acts as an additional design parameter which can be used to improve the behavior of the event-triggered control loop, e.g, by enlarging the region of stability. As the anti-windup mechanism only affects the controller structure, it has no influence on the lower bound $\bar{T}_{d}$ on the minimum inter-event $T_{\min }$ due to the limitations of the plant input $\tilde{\boldsymbol{u}}(t)$ (see Section 3.5). 


\section{Evaluation}

\subsection{Determination of the region of stability}

The stability conditions presented in this article include the linear decision variables $\boldsymbol{W}, \boldsymbol{S}, \boldsymbol{Z}$ and $\eta$ if the parameters $\tau_{i}$ are fixed. $\boldsymbol{R}$ and $\delta$ are either a priori given or additional decision variables depending on the objective of the algorithm (Tarbouriech et al. 2011). The following algorithm has been used to determine the regions of stability within this section:

1. Given $\boldsymbol{R}$ and $\delta$, choose a suitable objective function $f(\mathcal{E}(\boldsymbol{P}, \eta))$.

2. Fix $\tau_{1}, \tau_{2}, \tau_{3}$ and $\tau_{4}$.

3. Solve for $\boldsymbol{W}, \boldsymbol{S}, \boldsymbol{Z}$ and $\eta$ the optimization problem

$\min \{f(\mathcal{E}(\boldsymbol{P}, \eta))\}$

subject to the inequalities of either Theorem 1, Corollary 1 or Corollary 3.

In order to execute this algorithm, a suitable size criterion has to be found for maximizing the respective region of stability. There are several possibilities that translate into different objective functions $f(\mathcal{E}(\boldsymbol{P}, \eta)$ ) (see Tarbouriech et al. 2011). Two examples are:

- $\quad f(\mathcal{E}(\boldsymbol{P}, \eta))=n \log (\eta)+\log (\operatorname{det}(\boldsymbol{P}))$ referring to volume maximization.

- $f(\mathcal{E}(\boldsymbol{P}, \eta))=\beta_{0} \eta+\beta_{1} \operatorname{trace}(\boldsymbol{P})$ with weighting parameters $\beta_{0}$ and $\beta_{1}$ which leads to ellipsoids that are homogeneous in all directions.

However, the objective functions above become nonlinear due to the transformation $\boldsymbol{P}=\boldsymbol{W}^{-1}$. To avoid this, the optimization problem

$$
\begin{aligned}
& \min \{\operatorname{trace}(-\boldsymbol{W})\} \\
& \text { subject to inequalities Eqs. } 16-18
\end{aligned}
$$

has been used in the following evaluation which was solved by means of the YALMIP toolbox (Löfberg 2004).

Note that the outcome of the optimization might severely depend on the selection of the parameters $\tau_{i}$. Therefore, in order to obtain the maximum region of stability, a grid of corresponding $\tau_{i}$ needs to be defined based on which the optimization has to be carried out for every grid point.

\subsection{Simulations}

The simulation results are borrowed from Lehmann et al. (2012), where the unstable scalar system

$$
\dot{x}_{p}(t)=0.1 x_{p}(t)+\tilde{u}(t), \quad x_{p}(0)=x_{p 0}
$$

has been considered which is controlled by the event-triggered PI controller

$$
\begin{gathered}
\dot{x}_{c}(t)=x_{p}\left(t_{k}\right), \quad x_{c}\left(t_{k}\right)=x_{c k} \\
u(t)=-x_{c}(t)-1.6 x_{p}\left(t_{k}\right)
\end{gathered}
$$


$\left(t \in\left[t_{k}, t_{k+1}\right), w(t)=d(t)=0\right)$. The state information $x_{p}\left(t_{k}\right)$ is sent from the event generator to the controller whenever the event condition

$$
|e(t)|=\left|x\left(t_{k}\right)-x(t)\right|=\bar{e}
$$

is met. Considering the definition of the general event condition 7, this event condition results by setting $\boldsymbol{R}=1$ and $\delta=\bar{e}^{-2}$. Furthermore, the actuator output $\tilde{u}(t)$ is subject to limitations according to $\tilde{u}(t)=\operatorname{sat}(u(t))$ with $u_{0}=0.4$ (see Eq. 4 ).

Behavior of the event-triggered control loop Figure 4 shows the stability regions for different values of the event threshold $\bar{e}$ and $\tau_{1}=\tau_{2}=0.1$. The figure indicates that the size of the region decreases by increasing the event threshold, where $\bar{e}=0.15$ denotes the maximum threshold for which the inequalities 16-18 yield a feasible solution. The region obtained for $\bar{e}=0.15$ is denoted in the following by $\mathcal{E}(0.15)$.

The behavior of the event-triggered PI-control loop is depicted in Fig. 5. Here, the upper plot shows the plant state $x_{p}(t)$. The controller state $x_{c}(t)$ is depicted in the middle plot. Three different scenarios are considered.

The behavior using $\bar{e}=0.15$ with $\boldsymbol{x}_{0} \in \mathcal{E}(0.15)$ is drawn by the solid lines. As expected, the behavior remains stable. The dashed lines indicate the behavior obtained by starting from the same initial condition $\boldsymbol{x}_{0}$ but with an increased event threshold $(\bar{e}=0.9)$. The behavior becomes unstable. The same holds by choosing an admissible event threshold $\bar{e}=0.15$ but considering an initial state $\boldsymbol{x}_{0} \notin \mathcal{E}(0.15)$ $\left(\boldsymbol{x}_{0}=\left[\begin{array}{ll}1.9 & 0.5\end{array}\right]^{T}\right)$ depicted by the dotted lines. In the latter two cases the instability results from the fact that the input signal $\tilde{u}(t)$ is kept almost always in its saturation bounds (see lower plot of the figure) which is caused by a large controller state $x_{c}(t)$ resulting from integrator windup.

Anti-windup compensation Figure 6 shows how the anti-windup extension affects the region of stability. Choosing

$$
K_{A W}=-2,
$$

Fig. 4 Stability regions for different event thresholds; $\bar{e}=0$ indicates the region of stability for the corresponding continuous-time PI-control loop

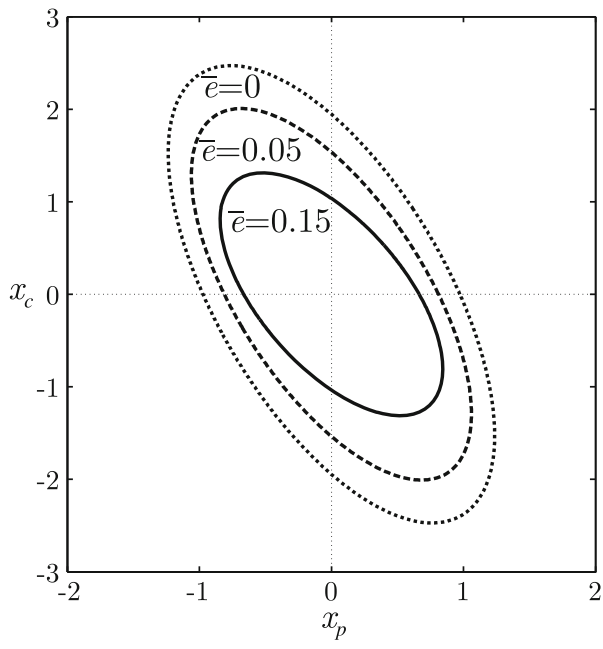


Fig. 5 Behavior of the event-triggered PI-control loop for: $1 . \bar{e}=0.15$, $\boldsymbol{x}_{0} \in \mathcal{E}(0.15)$ (solid lines), 2 . $\bar{e}=0.9, x_{0} \in \mathcal{E}(0.15)$ (dashed lines $), 3 . \bar{e}=0.15, \boldsymbol{x}_{0} \notin \mathcal{E}(0.15)$ (dotted lines)
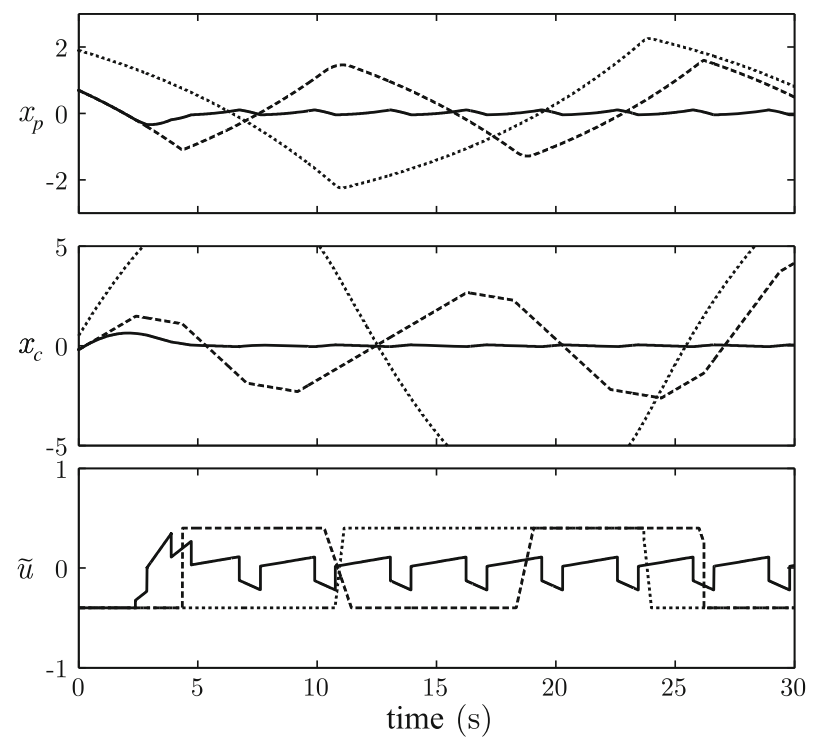

the region is significantly increased and even includes the region obtained for continuous-time PI control without compensation.

Moreover, Fig. 7 compares the resulting trajectories obtained with and without anti-windup control $\left(K_{A W}=-2\right)$ for $\boldsymbol{x}_{0} \in \mathcal{E}(0.15)$ and $\bar{e}=0.15$. It is shown that the overshoot with anti-windup control is much smaller compared to the scheme without compensation (upper plot) due to a much smaller controller state (second plot). This leads to a significant shorter period in which the actuator is saturated which can be seen at the beginning of the simulation (third plot). The lower plot of Fig. 7 shows the event times. The anti-windup control (AW) decreases the overall number of events

Fig. 6 Increase of the stability region due to anti-windup compensation

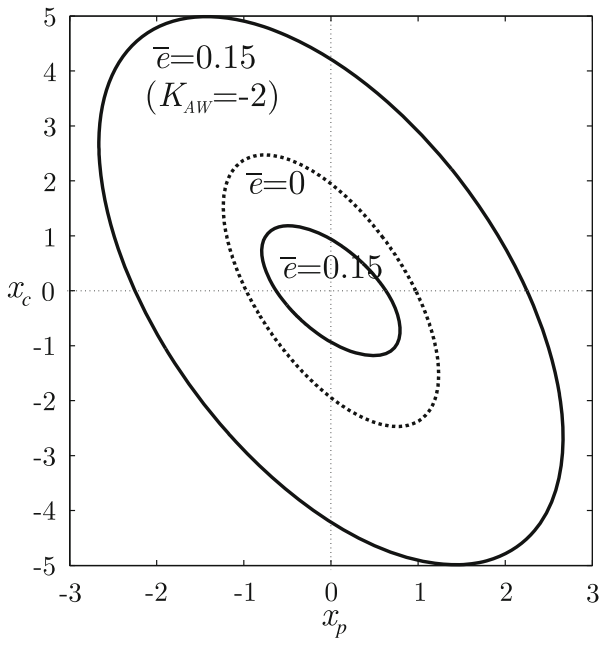


Fig. 7 Behavior of the event-triggered PI-control loop with (dotted lines) and without (solid lines) anti-windup compensation

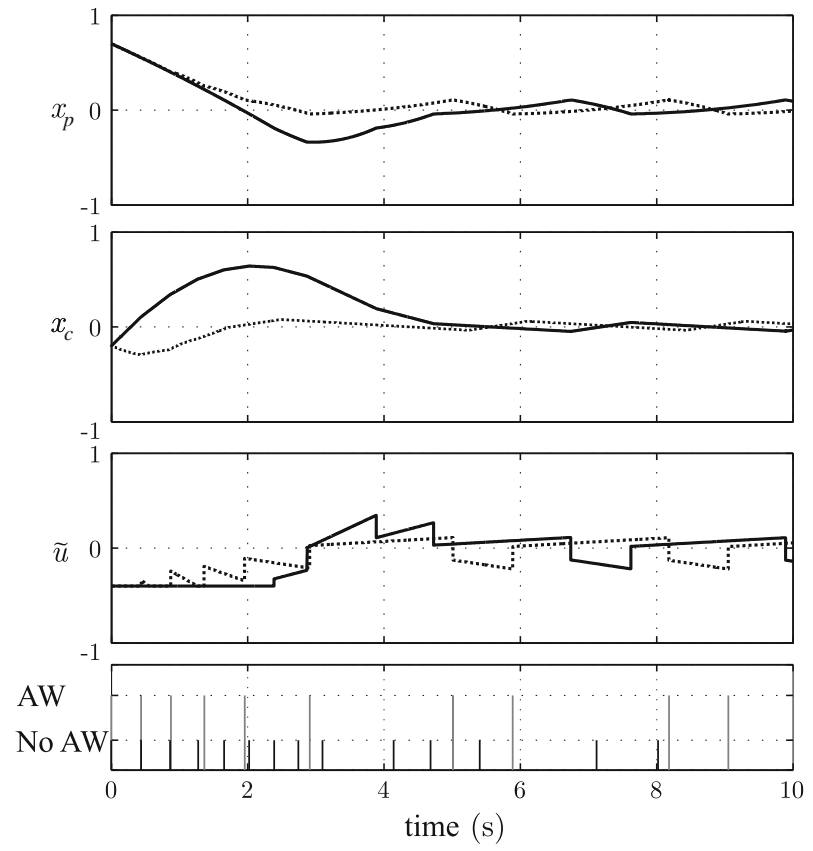

(9 instead of 13 events in the time interval considered) primarily by reducing the communication during the transient behavior.

\subsection{Experiments}

For the experimental evaluation a first-order tank system controlled by an eventtriggered PI-controller has been used. The experimental setup is depicted in Fig. 8. The central component of the experiment is a cylindrical tank whose inflow can be controlled by a pump with $\tilde{u} \in[0,12] \mathrm{V}$. The plant state $x_{p}(t)$ refers to the water

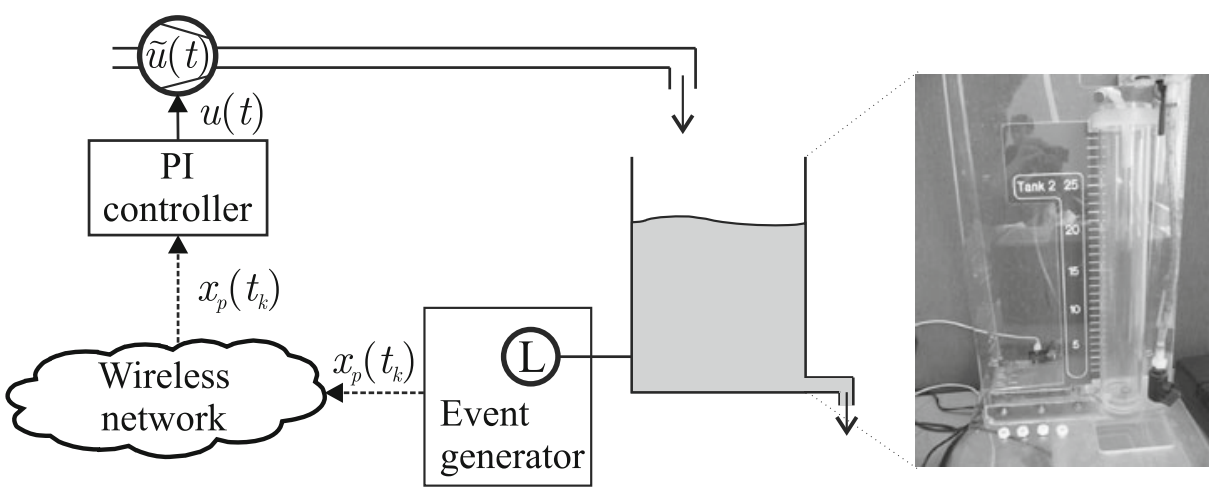

Fig. 8 Experimental setup 
level within the tank which can be continuously measured by a pressure sensor. Moreover, the outflow of the tank depends on the current water level. The node incorporating the event generator has access to the current measurements and uses a wireless communication network to send new information to the controller. The controller and the actuator are connected by wire.

As such a tank system usually possesses nonlinear dynamics (see Lehmann and Lunze 2011), the system has been linearized around the operating point

$$
x_{\mathrm{OP}}=10 \mathrm{~cm}, \quad \tilde{u}_{\mathrm{OP}}=5.43 \mathrm{~V}
$$

which leads to the stable linear state-space model

$$
\partial \dot{x}_{p}(t)=-\frac{1}{15} \partial x_{p}(t)+\frac{4}{15} \partial \tilde{u}(t), \quad \partial x_{p}(0)=\partial x_{p 0}
$$

with $\partial x_{p}(t)=x_{p}(t)-x_{\mathrm{OP}}$ and $\partial \tilde{u}(t)=\tilde{u}(t)-\tilde{u}_{\mathrm{OP}}$.

The event-triggered PI controller that controls the tank is given by

$$
\begin{aligned}
\dot{x}_{c}(t) & =\partial x_{p}\left(t_{k}\right)+K_{A W}(\tilde{u}(t)-u(t)), \quad x_{c}\left(t_{k}\right)=x_{c k} \\
\partial u(t) & =-0.1 x_{c}(t)-\partial x_{p}\left(t_{k}\right)
\end{aligned}
$$

$\left(t \in\left[t_{k}, t_{k+1}\right)\right)$ which gets the state information $\partial x_{p}\left(t_{k}\right)$ from the event generator whenever event condition 44 is satisfied.

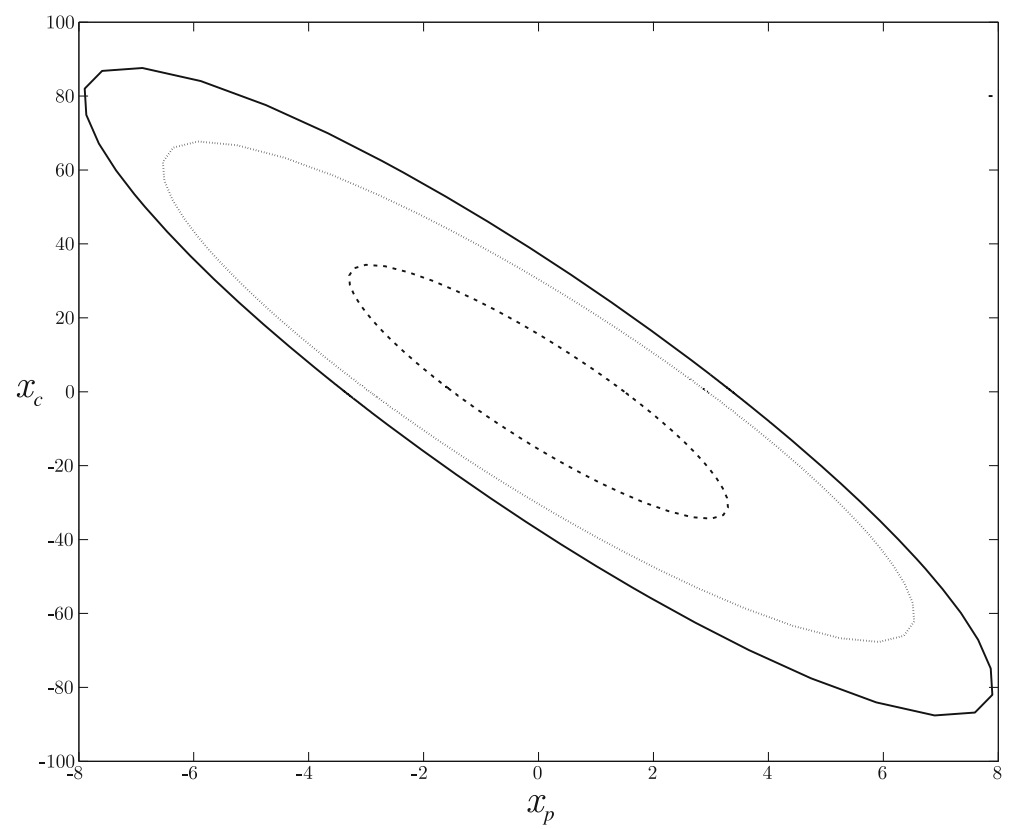

Fig. 9 Stability regions for $\bar{e}=1$ and $u_{0}=1$ (solid line), $\bar{e}=2$ and $u_{0}=1$ (dotted line), and $\bar{e}=1$ and $u_{0}=0.5$ (dashed line) 
As the saturation limits are non-symmetric $(\tilde{u} \in[0,12] \mathrm{V})$ and in order to be able to adapt the bounds, the saturation limits are artificially set around the operating point $\tilde{u}_{\mathrm{OP}}=5.43 \mathrm{~V}$ according to

$$
\begin{aligned}
& u_{\max }=\tilde{u}_{\mathrm{OP}}+u_{0} \\
& u_{\min }=\tilde{u}_{\mathrm{OP}}-u_{0} .
\end{aligned}
$$

Behavior of the event-triggered control loop Figure 9 shows the stability regions obtained for different event thresholds $\bar{e}$, different values of the saturation limit $u_{0}$ and no anti-windup compensation $\left(K_{A W}=0\right)$. The figure illustrates that, as expected, both increasing the event threshold $\bar{e}$ and reducing the saturation bound $u_{0}$ decrease the region of stability.

The behavior of the event-triggered control loop with $x_{p}(0)=5 \mathrm{~cm}$ and $x_{c}(0)=0$ and no anti-windup compensation is depicted in Fig. 10. The upper plot depicts the plant state $x_{p}(t)$ (circles and stars indicate the event times), the middle plot shows the controller state $x_{c}(t)$ and the plant input $\tilde{u}(t)$ is drawn in the lower plot.

It can be seen that by using $u_{0}=1$ and $\bar{e}=1$, the behavior of the event-triggered control loop remains stable. In fact, since the plant is stable and the input is subject to saturation, it is not possible to obtain an unbounded increase of the plant state $x_{p}(t)$.

However, by setting the saturation limit to $u_{0}=0.1$ and the event threshold to $\bar{e}=2$, an unstable behavior of the controller state $x_{c}(t)$ can be observed (dashed lines). This behavior is caused by the sticking effect (Tiberi et al. 2012; Vasyutynskyy
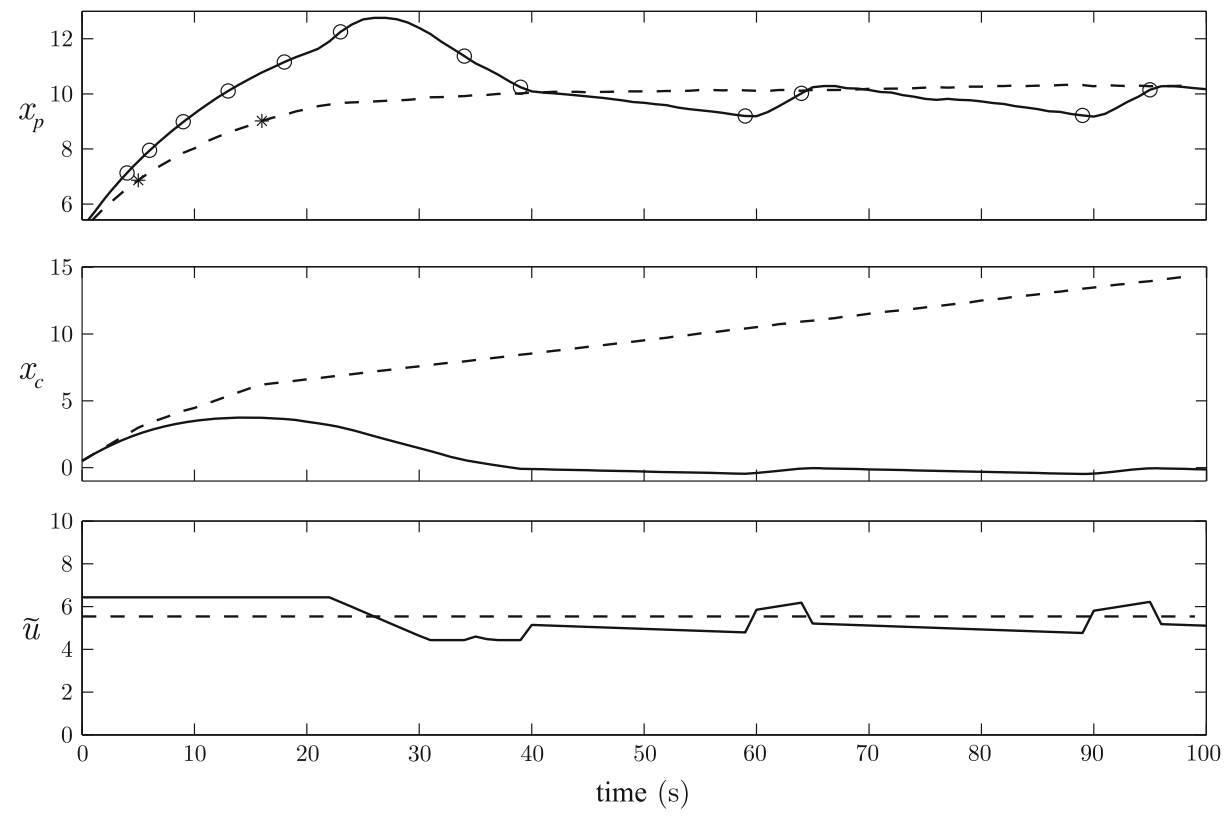

Fig. 10 Behavior of the event-triggered control loop for $u_{0}=1, \bar{e}=1$ (solid lines) and $u_{0}=0.1, \bar{e}=$ 2 (dashed lines) 


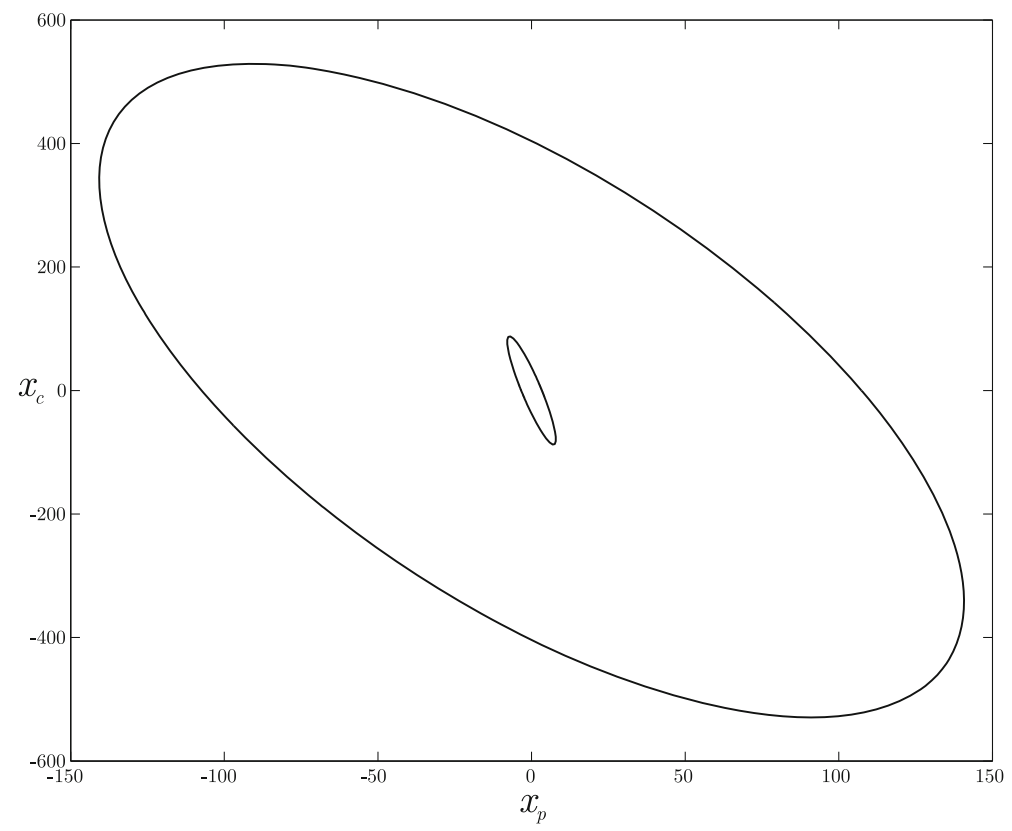

Fig. 11 Stability regions for $\bar{e}=1, u_{0}=1$ with (large region) and without (small region) anti-windup compensation
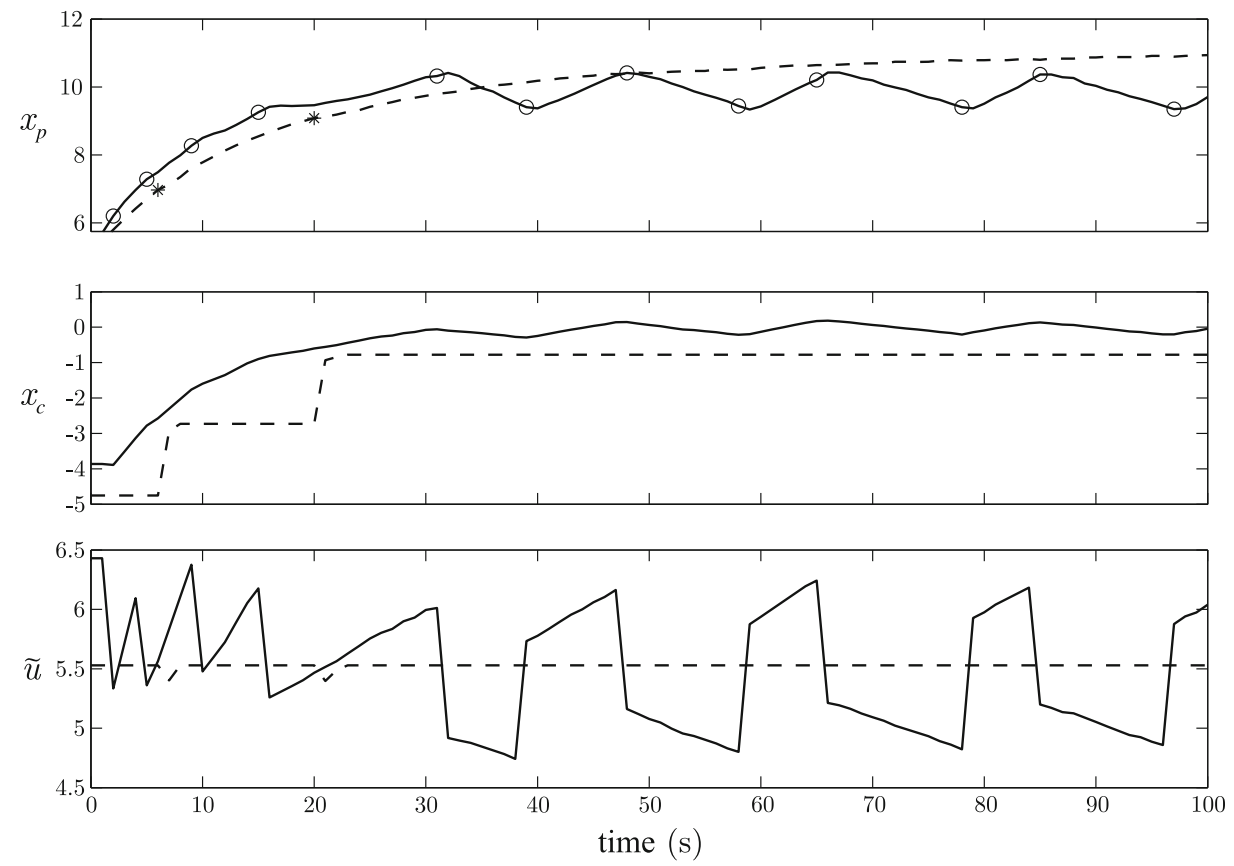

Fig. 12 Behavior of the event-triggered control loop for $u_{0}=1, \bar{e}=1$ (solid lines) and $u_{0}=0.1, \bar{e}=$ 2 (dashed lines) both with anti-windup compensation and gain $K_{A W}=-1$ 
and Kabitzsch 2006), where, in this case, the severe actuator saturation avoids further events after the second event time (indicated by the second star in the upper plot of the figure). As the state information used by the controller deviates from the operating point at that time instance, the controller state $x_{c}(t)$ increases monotonically for all future times.

Anti-windup compensation Figures 11 and 12 show the effect of anti-windup compensation with gain $K_{A W}=-1$ on the behavior of the event-triggered control loop.

It is clearly demonstrated that anti-windup compensation improves both, the region of stability (see Fig. 11) and the behavior of the event-triggered control loop (see Fig. 12) in terms of a better transient behavior (solid lines) and a stable integrator state (dashed lines). In contrast to the simulation results no decrease of the information exchange can be obtained which might be caused by model uncertainties leading to slightly different plant dynamics in the experiments.

In the simulation and the experiment an event-triggered PI controller has been used to control a first-order plant whose state is measurable. These rather simple examples have been chosen to demonstrate the consequences of actuator saturation in an illustrative and clear way. However, remember that the method proposed in this article can be more generally applied to linear plants of arbitrary order controlled by a general dynamic output feedback controller.

\section{Conclusions}

In this article, the influence of actuator saturation on event-triggered control has been addressed. As a main result LMI conditions have been derived which allow to determine regions of stability for the event-triggered closed-loop system. The results have been extended by incorporating a static anti-windup mechanism. It has been shown that in both cases the information exchange over the feedback link is bounded.

Simulations and experimental results with a wirelessly controlled tank system have validated the theoretical results and showed that by using anti-windup compensation the performance of the event-triggered control loop can be significantly improved in terms of larger regions of stability and a better overall performance.

Future work will include alternative methods for deriving the stability regions, the design of event-triggered anti-windup mechanisms and the consideration of communication imperfections.

\section{References}

Anta A, Tabuada P (2010) To sample or not to sample: self-triggered control for nonlinear systems. IEEE Trans Automat Contr 55(9):2030-2042 
Årzén KE (1999) A simple event-based PID controller. In: Proceedings of IFAC world congress, Beijing, pp 423-428

Åström KJ, Bernhardsson B (1999) Comparison of periodic and event based sampling for first-order stochastic systems. In: Proceedings of IFAC world congress, Beijing, pp 301-306

Åström KJ, Hägglund T (1995) PID controllers: theory, design, and tuning, ISA

Baillieul J, Antsaklis P (2007) Control and communication challenges in networked real-time systems. Proc IEEE 95(1):9-28

Bemporad A, Heemels WPMH, Johansson M (2010) Networked control systems. In: Lecture notes in control and information sciences. Springer

Blanchini F (1999) Set invariance in control. Automatica 35(11):1747-1767

Cassandras CG, Lafortune S (2008) Introduction to discrete event systems. Springer, New York

Cervin A, Henningsson T (2008) Scheduling of event-triggered controllers on a shared network. In: Proceedings of IEEE conference on decision and control, Cancun, pp 3601-3606

Donkers MCF, Heemels WPMH (2010) Output-based event-triggered control with guaranteed $\mathcal{L}_{\infty^{-}}$gain and improved event-triggering. In: Proceedings of IEEE conference on decision and control, Atlanta

Heemels WPMH, Gorter RJA, van Zijl A, van den Bosch PPJ, Weiland S, Hendrix WHA, Vonder MR (1999) Asynchronous measurement and control: a case study on motor synchronization. Control Eng Pract 7(12):1467-1482

Heemels WPMH, Sandee J, van den Bosch P (2008) Analysis of event-driven controllers for linear systems. Int J Control 81(4):571-590

Lehmann D (2011) Event-based state-feedback control. Logos Verlag, Berlin

Lehmann D, Johansson KH (2012) Event-triggered PI control subject to actuator saturation. In: Proceedings of IFAC conference on advances in PID control, Brescia

Lehmann D, Kiener GA, Johansson KH (2012) Event-triggered PI control: saturating actuators and anti-windup compensation. In: IEEE conference on decision and control (accepted)

Lehmann D, Lunze J (2011) Extension and experimental evaluation of an event-based statefeedback approach. Control Eng Pract 19(2):101-112

Löfberg J (2004) YALMIP: a toolbox for modeling and optimization in MATLAB. In: Proceedings of the CACSD conference, Taipei

Lunze J, Lamnabhi-Lagarrigue F (2009) Handbook of hybrid systems control. Cambridge University Press, Cambridge, UK

Lunze J, Lehmann D (2010) A state-feedback approach to event-based control. Automatica 46(1):211-215

Nair GN, Fagnani F, Zampieri S, Evans RJ (2007) Feedback control under data rate constraints: an overview. Proc IEEE 95(1):108-137

Otanez PG, Moyne JG, Tilbury DM (2002) Using deadbands to reduce communication in networked control systems. In: Proceedings of American control conference, Anchorage, pp 3015-3020

Tabuada P (2007) Event-triggered real-time scheduling of stabilizing control tasks. IEEE Trans Automat Contr 52(9):1680-1685

Tarbouriech S, Garcia G, Gomes da Silva Jr JM, Queinnec I (2011) Stability and stabilization of linear systems with saturating actuators. Springer, London

Tarbouriech S, Prieur C, Gomes da Silva Jr JM (2006) Stability analysis and stabilization of systems presenting nested saturations. IEEE Trans Automat Contr 51(8):1364-1371

Tiberi U, Araújo J, Johansson KH (2012) On event-based PI control of first-order processes. In: Proceedings of IFAC conference on advances in PID control, Brescia

Tipsuwan Y, Chow MY (2003) Control methodologies in networked control systems. Control Eng Pract 11:1099-1111

Vasyutynskyy V, Kabitzsch K (2006) Implementation of PID controller with send-on-delta sampling. In: Proceedings of international control conference, Glasgow

Wang X, Lemmon MD (2009) Self-triggered feedback control systems with finite-gain $\mathcal{L}_{2}$ stability. IEEE Trans Automat Contr 54(3):452-467

Zaccarian L, Teel AR (2011) Modern anti-windup synthesis: control augmentation for actuator saturation, Princeton

Zhang W, Branicky MS, Philips SM (2001) Stability of networked control systems. IEEE Control Syst Mag 21(1):84-99 


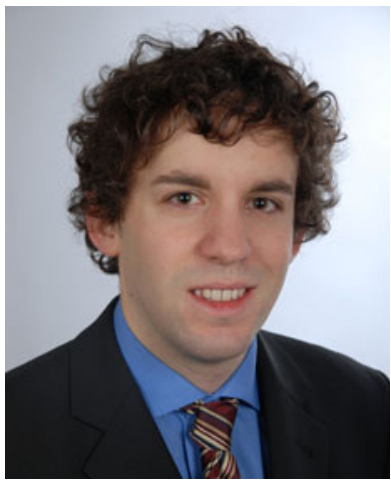

Georg Alexander Kiener received his diploma (equivalent to MSc) in Electrical Engineering from the Technische Universität München (TUM) and an Honours Degree in Technology Management from the Center of Digital Technology and Management (CDTM) in 2012. Both institutes are located in Munich, Germany. He was a visiting student researcher at the University of California at Berkeley and at the Royal Institute of Technology (KTH) in Stockholm, Sweden.

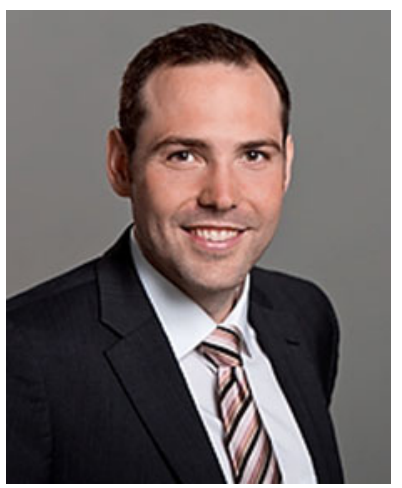

Daniel Lehmann received his Diploma (equivalent to MSc) in Electrical Engineering and his Ph.D. degree in Automatic Control from the Ruhr-University Bochum (Germany) in 2007 and 2011, respectively. Since then, he is a postdoctoral researcher at the Automation Control Laboratory and the ACCESS Linnaeus Centre at the Royal Institute of Technology (KTH), Sweden. His research interests include analysis and design of event-based controlled systems, networked control systems, PID control, wireless control, systems subject to actuator saturation and hybrid control. 


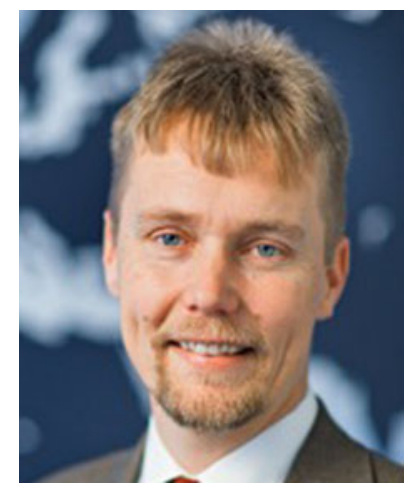

Karl Henrik Johansson is Director of the KTH ACCESS Linnaeus Centre and Professor at the School of Electrical Engineering, Royal Institute of Technology, Sweden. He is a Wallenberg Scholar and has held a Senior Researcher Position with the Swedish Research Council. He received MSc and $\mathrm{PhD}$ degrees in Electrical Engineering from Lund University. He has held visiting positions at UC Berkeley (1998-2000) and California Institute of Technology (2006-2007). His research interests are in networked control systems, hybrid and embedded control, and control applications in automotive, automation and communication systems. He was a member of the IEEE Control Systems Society Board of Governors 2009 and the Chair of the IFAC Technical Committee on Networked Systems 2008-2011. He has been on the Editorial Boards of Automatica (2003-2006) and IEEE Transactions on Automatic Control (2008-2010), and is currently on the Editorial Boards of IET Control Theory and Applications and the International Journal of Robust and Nonlinear Control. He was the General Chair of the ACM/IEEE Cyber-Physical Systems Week (CPSWeek) 2010 in Stockholm. $\mathrm{He}$ has served on the Executive Committees of several European research projects in the area of networked embedded systems. In 2009, he received the Best Paper Award of the IEEE International Conference on Mobile Ad-hoc and Sensor Systems. He was awarded an Individual Grant for the Advancement of Research Leaders from the Swedish Foundation for Strategic Research in 2005. He received the triennial Young Author Prize from IFAC in 1996 and the Peccei Award from the International Institute of System Analysis, Austria, in 1993. He received Young Researcher Awards from Scania in 1996 and from Ericsson in 1998 and 1999. 